\title{
EchoGéo
}

40 | 2017

Ressources urbaines (2)

\section{Mobilité internationale étudiante et mobilité sociale : ressources et liens sociaux}

\section{Carolina Pinto-Baleisan and Matthieu Delage}

\section{(2) OpenEdition \\ 1 Journals}

\section{Electronic version}

URL: https://journals.openedition.org/echogeo/14989

DOI: 10.4000/echogeo.14989

ISSN: 1963-1197

\section{Publisher}

Pôle de recherche pour l'organisation et la diffusion de l'information géographique (CNRS UMR 8586)

\section{Electronic reference}

Carolina Pinto-Baleisan and Matthieu Delage, "Mobilité internationale étudiante et mobilité sociale : ressources et liens sociaux", EchoGéo [Online], 40 | 2017, Online since 30 June 2017, connection on 11 August 2021. URL: http://journals.openedition.org/echogeo/14989 ; DOI: https://doi.org/10.4000/ echogeo.14989

\section{This text was automatically generated on 11 August 2021}

EchoGéo est mis à disposition selon les termes de la licence Creative Commons Attribution - Pas d'Utilisation Commerciale - Pas de Modification 4.0 International (CC BY-NC-ND) 


\title{
Mobilité internationale étudiante et mobilité sociale : ressources et liens sociaux
}

\author{
Carolina Pinto-Baleisan and Matthieu Delage
}

1 Le livre dont il est ici question est la publication de la thèse de Carolina Pinto-Baleisan, soutenue en 2013 en France: Pinto-Baleisan Carolina, 2017. Migrations étudiantes sudaméricaines, trajectoires sociales et bifurcations biographiques. Paris, La Documentation française, collection Études et recherches.

2 Le travail de recherche qu'elle a mené pendant cinq ans sur deux terrains de grandes métropoles universitaires en France et aux États-Unis portait sur le lien entre mobilité spatiale et mobilité sociale dans les mobilités internationales étudiantes.

3 L'entretien, conduit par Mathieu Delage, a été réalisé à distance (Paris-Valparaiso) le 28 juin 2017.

4 - Matthieu Delage (MD). Pourriez-vous en quelques mots présenter la recherche qui a donné lieu à ce livre?

5 - Carolina Pinto-Baleisan (CPB). Cet ouvrage se propose d'explorer le vécu des jeunes adultes d'origine chilienne et colombienne au cours des études, au niveau master ou doctorat, loin du pays d'origine. Il vise à comprendre les décisions prises à la fin de cette expérience à la fois individuelle et sociale : poursuivre le projet fixé au départ, l'abandonner, le développer ou le changer? L'intérêt porte sur les transformations internes de l'individu migrant, accentuées par la distance physique, sociale et culturelle vis-à-vis de la société d'origine. La démarche scientifique se fonde sur une approche compréhensive et biographique. La population d'étude se compose d'étudiants d'origine chilienne ou colombienne inscrits en troisième cycle - en master 2 ou en doctorat - n'ayant pas la nationalité du pays de résidence, en l'occurrence la France ou les États-Unis. Ils habitent et poursuivent des études dans les villes de Paris, New York ou Boston. Le choix de cette comparaison permet, d'une part, de saisir la concurrence internationale entre métropoles universitaires entre la France et les États-Unis et, 
d'autre part, de s'intéresser à deux pays d'origine dits « émergents » où s'est constituée une classe moyenne nombreuse et qui offrent des profils étudiants variés.

- MD. La question des ressources se pose de manière cruciale pour les étudiants étrangers, à Paris aussi bien qu'à Boston. Vous avez été attentive à l'accès au logement, et vous montrez que les liens sociaux constituent une ressource aussi importante que l'offre institutionnelle du logement étudiant. De quelle manière les étudiants que vous avez enquêtés mobilisent-ils ces ressources sociales?

7 - CPB. Se loger est un impératif dans tout projet migratoire. Les solutions d'hébergement pour les étudiants étrangers sont variées : vivre dans une résidence universitaire, louer un appartement individuel ou être logé chez l'habitant n'en sont que quelques-unes. Ces alternatives sont saisies d'une manière différente selon l'offre de chaque ville et les ressources dont les étudiants disposent. Par exemple, un étudiant qui est boursier de la coopération française peut raisonnablement prétendre à vivre dans une résidence universitaire du CROUS à Paris. Mais pour ceux et celles qui autofinancent leurs études, qui sont les plus nombreux, avoir des connaissances sur place est fondamental pour trouver plus facilement une offre dans le marché locatif privé. J'observe donc la puissance des réseaux sociaux transnationaux pour favoriser l'insertion résidentielle. Il y a un bouche-à-oreille, des sites Internet avec des astuces, des solidarités particulières entre étudiants et résidents de la même origine, autant en France qu'aux États-Unis. À ce sujet, la comparaison montre que les Colombiens ont bien plus de ressources transnationales que les Chiliens, vu que $10 \%$ de la population colombienne habite à l'étranger. Il est donc très fréquent que les étudiants connaissent depuis le tout début du séjour quelqu'un dans la ville de destination. Cette personne peut leur offrir un hébergement temporaire ou une opportunité pour commencer un boulot. Pour réussir l'installation dans une ville étudiante étrangère, être en contact avec des réseaux amicaux et de connaissances peut être aussi important que d'avoir des ressources économiques.

Un premier réseau à explorer est la famille. Selon l'enquête sur les étudiants universitaires en France, menée par l'observatoire de la vie étudiante, 10,6\% des étudiants non européens sont logés chez des membres de leur famille, une partie beaucoup plus importante que celle des étudiants français $(1,6 \%)$ ou celle des européens $(2,1 \%)$. Ces résultats reflètent principalement la situation des étudiants provenant des pays des ex-colonies françaises car les étudiants latino-américains ayant de la famille en France sont moins nombreux. Un deuxième réseau social qui renvoie à la société d'origine est celui des exilés politiques. C'est le cas de Tadeo, par exemple, étudiant chilien de master vivant en couple avec une étudiante chilienne comme lui dans le XVIII arrondissement. Ils ont été reçus temporairement chez un couple d'amis de leurs parents qui, exilés en France depuis la période de la dictature militaire au Chili, sont devenus une sorte de "faux parents" pour le jeune couple. Ce type de réseau opère souvent dans une logique communautaire où l'étudiant a une place ambiguë car il partage la même nationalité mais n'affronte pas les mêmes conditions de séjour. Pour des raisons historiques, ces réseaux sont disponibles plus fréquemment en France qu'aux États-Unis. Un dernier réseau à mentionner, qui renvoie à la position sociale de l'étudiant dans son pays, sont les communautés d'anciens étudiants du pays d'origine. Par exemple, un réseau très vigoureux décrit par certains étudiants venus aux ÉtatsUnis est celui des anciens étudiants de l'université Catholique au Chili ou de l'Universidad de Los Andes en Colombie, qui permettent d'élargir les interconnaissances 
depuis le premier jour à l'étranger. Ces réseaux d'étudiants fonctionnent à l'égard du logement en organisant l'information d'une manière directe (bouche-à-oreille) ou indirecte (associations d'étudiants, sites internet spécialisés, etc.) entre ses membres. Je remarque finalement que les étudiants partis en France, qui affrontent le plus de difficultés au niveau de logement, font plus souvent appel à ces réseaux que les étudiants à destination des États-Unis, car une bonne partie d'entre eux peuvent accéder à des offres proposées par leurs universités.

9 - MD. De même, obtenir un visa, organiser son voyage, prolonger son séjour au-delà de la fin des études s'avèrent un parcours du combattant et, encore une fois, la manière d'activer des liens sociaux se révèle être la meilleure, sinon la seule solution.

10 - CPB. Oui, en effet, mais c'est important de distinguer trois moments différents car les liens sociaux mobilisés à chaque étape ne sont pas les mêmes. Le premier moment concerne la période avant de partir, la préparation du séjour, qui se déroule dans le pays d'origine. Lorsqu'un jeune diplômé universitaire se rend aux services consulaires pour demander un visa étudiant et partir en France ou aux États-Unis, les dispositifs institutionnels et juridiques jouent un rôle très important. Tout d'abord, le premier filtre concerne les établissements d'éducation supérieure. Ayant la mission d'accueillir les meilleurs candidats pour assurer leur propre rayonnement, les établissements français et américains utilisent différentes procédures. Pour certains candidats, des amitiés et des connaissances transnationales peuvent aider à préparer leurs candidatures pour les universités. Même si ce sont bien les institutions éducatives qui permettent de démarrer une demande de visa auprès d'un consulat français ou nordaméricain à l'étranger, leur action est pourtant subordonnée aux politiques migratoires nationales. Dans ce sens-là, il est devenu plus difficile d'être un étudiant étranger car les conditions d'un visa étudiant semblent de plus en plus sélectives, privilégiant la poursuite d'études à partir du niveau de master et la justification de ressources économiques. Pendant les démarches administratives, des liens sociaux forts comme la famille permettent de contourner certaines formalités grâce à une attestation de prise en charge signée généralement par les parents, ou bien grâce au transfert d'un montant d'argent assez élevé sur le compte bancaire de l'étudiant.

11 Le deuxième moment où j'observe ce "parcours du combattant» tient à la prolongation du séjour d'études dans le pays d'accueil. Tout changement de parcours d'études ainsi qu'une prolongation en doctorat doivent être validés par les autorités migratoires et peuvent conduire à des refus ou à des changements de statut juridique pour les étudiants. Les intéressés doivent donc faire encore preuve de solvabilité économique, démontrant qu'ils peuvent couvrir le paiement des frais de scolarité et leur entretien ainsi que celui de leur famille. Pour ce faire, ceux qui ont une situation financière plus fragile activent des liens sociaux variés : la famille dans le pays d'origine ainsi bien que des amitiés sur place peuvent aider à collecter une somme d'argent sur un compte bancaire, somme qui, après l'entretien, est rendue à ses propriétaires. Cependant cette stratégie fonctionne surtout en France, puisque les montants pour étudier aux États-Unis sont tellement élevés que souvent la seule solution pour prolonger les études est d'obtenir une bourse ou une allocation doctorale.

12 Enfin, un troisième moment de difficulté qui peut être surmonté grâce aux liens sociaux tient au changement de statut, c'est-à-dire le passage juridique de l'étudiant à l'immigrant. L'accès à l'emploi hautement qualifié est organisé à travers l'attribution de permis de résidence temporaires qui autorisent (sous de nombreuses conditions) 
l'exercice professionnel des étrangers. Comme pour tous les travailleurs, la construction d'un réseau professionnel et amical est crucial pour réussir à trouver un emploi et obtenir un premier contrat d'emploi (Granovetter, 1973). Les migrations de scientifiques semblent en principe être les plus désirées par les pays développés, leur accordant l'assouplissement de certaines conditions pour les attirer. Pourtant, ma recherche ne permet pas de conclure que des politiques favorables aux diasporas scientifiques soient effectives. Une autre voie d'accès au changement de statut relève de la formation de couples. Au regard du droit à une vie familiale, les cadres juridiques des deux pays analysés procurent des autorisations qui accordent un accès sans restrictions au marché de l'emploi local. Toutefois, plusieurs contrôles doivent être franchis par les étudiants et leurs conjoints afin de faire reculer les soupçons de fraude. Cela dit, la formation de couples qui finit par un mariage ou une union civile est loin d'être la règle dans ces migrations étudiantes. Construire des liens amoureux forts nécessite plusieurs conditions, surtout de temps, ce qui n'est pas forcément le cas pour les étudiants en mobilité.

13 - MD. Il ressort de vos analyses que les déterminants sociaux, les milieux d'origine, les habitus de classe et les manières dont les rôles et les places sont assignés ou dont l'avenir est prédestiné constituent un facteur fondamental. Pourtant, vous montrez aussi que ces déterminants ne valent pas pour tous. Comme si «la règle du jeu» pouvait être réinventée en cours de route...

14 - $\mathrm{CPB}$. Le fait de se spécialiser à l'étranger témoigne d'attentes et de significations différentes selon les classes sociales. Certaines n'ont qu'un accès restreint à la mobilité et à la connaissance des pays étrangers, tandis que d'autres y sont au contraire familiarisées très tôt (Wagner, 2007). La dimension internationale apparaît comme un référent symbolique qui perpétue la tradition cosmopolite des classes supérieures. De la sorte, faire des études à l'étranger permet aux étudiants issus des couches favorisées de confirmer la maîtrise des codes sociaux demandés pour la reproduction des élites et d'obtenir à l'avenir plus assurément une place dominante. En revanche, pour les classes moyennes, la mise à distance de la hiérarchie sociale d'origine, qui se fait pendant le séjour d'étude, constitue une double opportunité d'ascension sociale. D'une part, le fait de devenir un étranger, c'est-à-dire un «homme sans passé » d'après Simmel (1979), permet de jouer avec les signes de distinction et de s'insérer autrement dans la société d'accueil. D'autre part, l'étudiant issu d'une classe moyenne peut prétendre au retour à des situations professionnelles qu'il considère plus élevées.

Bien entendu, seule une partie restreinte de la population qualifiée des régions " périphériques » poursuit un perfectionnement. Moins nombreux encore sont ceux qui le font à l'étranger. Considérant ainsi que l'espace international demeure hautement sélectif, cette recherche montre quelles sont les ressources nécessaires pour mettre en œuvre un tel projet et comment leur distribution inégale façonne et sélectionne les candidats depuis le départ. La reconstruction des backgrounds des personnes interrogées m'a conduite à adopter la notion de trajectoire afin d'explorer les biographies des jeunes en mobilité. Le concept de trajectoire vise aussi à restituer la capacité de l'individu à devenir sujet. Le devenir personnel est loin de se réduire aux héritages et à l'histoire personnelle. La trajectoire - métaphore empruntée à la balistique (Passeron, 1990) - dépend ainsi autant de l'impulsion de départ (combinant le poids du déterminisme de classe et de cycle de vie, et le déclencheur conjoncturel), qui donne la direction et l'orientation, que du changement intérieur personnel et 
subjectif (prise de distance, émergence d'un regard critique sur la société de départ, distension et renouvellement des liens, découverte d'une vocation pour un métier, etc.) qui amène au fur et à mesure à prendre autrement les décisions. Il est question alors de distinguer une logique de processus et de changement individuel qui déclenche, à chaque moment d'une trajectoire, d'autres événements supposés être fortuits. Certaines bifurcations ou changements de sens inattendus (Bessin et al., 2010, p. 397) se présentent pendant le déroulement de l'expérience de vie à l'étranger. Même si la plupart des étudiants ont une certaine idée sur ce qui les attend, ce type de séjour entraîne souvent une dose de surprise qui se traduit par une prise de distance avec soimême, avec le soi qui est resté au pays et qui d'une certaine manière s'est éclipsé. Très peu ont pu imaginer auparavant comment ils allaient vivre la distance. De même l'incertitude et les remises en question peuvent parfois se vivre violemment chez les jeunes adultes en mobilité à travers l'expérience de devoir "se serrer la ceinture ", de l'isolement, de la solitude, de se sentir classifié ou discriminé et d'autres difficultés variées. Certains abandonnent en cours de route, d'autres relativisent car l'horizon leur paraît meilleur. D'autres enfin vont se retrouver libres, apprécier de se passer de confort et de conformisme, plus encore pour ceux qui n'avaient pas encore de confort avant de partir. Ils arrivent à saisir des opportunités qui se présentent sur le moment et cette manière de prendre les décisions peut les conduire à prolonger leur séjour à l'étranger. Ces migrants font preuve d'une transformation interne dans leurs positionnements qui leur permet une priorisation des contraintes et un ajustement progressif des projets professionnels et migratoires.

16 Il faut cependant souligner que le changement interne de l'individu migrant ou cette capacité à se réinventer ne signifie pas l'annulation des contraintes structurelles ni des déterminismes sociaux. Une fois que les décisions cessent d'être soumises au projet initial, l'individu doit faire face à une autre sélectivité, celle de la migration hautement qualifiée. Les restrictions juridiques et le besoin de travailler pour préserver son indépendance s'imposent à l'individu avec leur force contraignante habituelle.

17 - MD. Ainsi, pour vous, la mobilité spatiale, qui semble une injonction pour les étudiants et les jeunes très qualifiés dans le monde et en particulier dans les pays émergents, c'est avant tout une affaire de mobilité sociale? Ou plutôt de reproduction sociale? Ce que vous dites des " étudiants encapsulés » est à ce titre particulièrement intéressant...

18 - CPB. Pour ceux et celles que j'appelle les « étudiants encapsulés » le séjour d'études à l'étranger est en quelque sorte prédisposé, soit par leur socialisation de classe, ou bien par leur milieu professionnel. Peu de surprises se présentent pour eux, les projets initiaux sont conservés en raison de déterminismes sociaux et institutionnels. Un exemple peut éclairer ce type de trajectoire. Manuela est une femme chilienne, ingénieure de formation, qui poursuit une spécialisation MBA au MIT. Même si cette femme se déclare très satisfaite de son expérience de vie à Boston et qu'un emploi lui a été récemment proposé, à la fin du programme, elle ne souhaite rien d'autre que retourner au Chili. D'une part, elle veut retourner dans son entreprise qui a financé une partie de sa formation et monter dans cette hiérarchie. D'autre part, son mari, lui aussi ingénieur, doit également reprendre son emploi. En conséquence, pas de changement. C'est surtout dans ce groupe qu'on peut parler de reproduction sociale car ce sont plusieurs générations qui ont été formées de cette manière. Les étudiants les plus privilégiés et membres des élites suivent une sorte de "couloir» pré-défini : il faut 
partir seulement pendant un ou deux ans et choisir certains programmes d'études dans quelques établissements précis (avant tout américains et historiquement liés aux élites comme l'université de Chicago, Harvard ou le MIT). En outre, la situation de famille doit faire preuve de soumission aux valeurs traditionnelles (peu de célibataires, jeunes couples mariés avant de partir, les femmes accompagnent les hommes qui étudient, etc.). Au retour dans le pays d'origine, la réussite est assurée pour eux, avec des emplois confirmés avant même d'arriver, pour continuer leur carrière ascendante vers des hauts postes de direction dans des entreprises, dans le monde politique ou bien dans les hauts fonctions de l'État.

19 D'autres individus, en revanche, les plus nombreux d'ailleurs, se situent dans des classes moyennes des pays émergents et, pour eux, la mobilité spatiale est un pari moins sûr. Pour certains, leur départ du pays d'origine peut être considéré comme un événement imprévu. Les dispositions, ils les ont, en termes de ressources symboliques et matérielles, mais ces jeunes professionnels concrétisent la décision de partir par le désir de changement qui a pu se révéler, par exemple, au gré d'un parcours professionnel difficile, ou bien à la suite d'une rupture affective. Pour d'autres dans ce même groupe, mais moins favorisés, le séjour d'études à l'étranger se construit dans l'espoir de gagner un outil méritocratique, un diplôme étranger, qui soit bien valorisé sur le marché de l'emploi car, jusqu'à présent, ils ont eu une insertion professionnelle difficile dans leur pays. Pour ces derniers, cette recherche de méritocratie n'exclut pas une émigration plus définitive. Au final, cette pratique en tant que moyen pour obtenir une ascension sociale dépend forcément d'où on se place, de la position sociale initiale. Une fois ce déterminant clair, toute aventure consistant à partir se spécialiser ailleurs implique une certaine prise de risque : rien n'est sûr. La plupart des étudiants partent sans savoir s'ils vont retourner au pays, notamment pour ceux qui cherchent à changer de métier : on perd ses contacts professionnels, on peut revenir avec un diplôme mais sans l'expérience nécessaire, donc il s'agit d'une expérience très incertaine. On peut être hautement diplômé et être cependant dans une situation très incertaine, c'est finalement le cas de la plupart des étudiants que j'ai enquêtés.

20 - MD. Il me semble que votre attention aux bifurcations de trajectoires qui sont finalement la clef de lecture la plus efficace pour comprendre les migrations internationales est un des apports fondamentaux de votre recherche.

21 - $\mathrm{CPB}$. Ma démarche analytique s'appuie largement dans la littérature française sur les trajectoires et les bifurcations. Ces notions combinées avec une analyse compréhensive des biographies éclairent le réagencement des déterminismes sociaux par les individus. Deux moments de prises de décision ont été étudiés : premièrement, comment les jeunes professionnels en sont venus à décider de suivre des études à l'étranger, et deuxièmement, comment ils sont amenés à revoir leur projet initial au cours du séjour. Je propose à cet effet une typologie qui synthétise la trajectoire socioprofessionnelle des interviewés avant le départ. Ensuite, j'observe à l'intérieur de chaque type l'évolution des projets professionnels et familiaux telle qu'elle s'exprime à la fin des études.

22 Je voudrais vous donner quelques exemples de bifurcations lisibles dans les biographies recueillies. Certaines interrogent le déterminisme de la mobilité sociale intergénérationnelle dans le pays d'origine. Il faut prendre en compte le fait que le Chili et la Colombie sont deux pays très inégalitaires où la mobilité sociale stagne de plus en plus pour les familles qui s'endettent pour voir leurs enfants devenir des diplômés 
d'université. Dans certains parcours éducatifs, le passage par un établissement scolaire ou universitaire prestigieux peut être le début d'une ascension sociale, du tissage d'un réseau de liens jusqu'alors inaccessibles et la mise en jeu de références croisées voire contradictoires (Lahire, 2001). L'entrée dans une institution d'éducation sélective, résultat combiné des succès scolaires et du soutien parental, permet à des enfants issus de milieux modestes ne disposant pas d'un capital social ou culturel international, d'accéder aux ressources et à l'imaginaire activant le désir de faire plus tard des études à l'étranger. Le choix de la discipline étudiée après la fin des études secondaires semble en revanche très conditionné par le milieu familial. Cependant, la décision de certains jeunes déjà actifs de reprendre des études à l'étranger est pour moi une bifurcation active et significative, en tant que décision consciente de changer de métier et de pallier certaines frustrations pendant le début de la vie adulte, notamment chez les enseignants du primaire et du secondaire qui font face à des salaires et des conditions de travail très peu gratifiants en Colombie ou au Chili. Faire des études à l'étranger est également une bifurcation active lorsque cette pratique permet une reconversion professionnelle, soit parce que le parcours précédemment suivi a été imposé par les parents contre le gré de l'étudiant, soit parce que le sentiment de stagnation professionnelle devient insupportable.

23 Enfin, j'observe aussi des bifurcations dans le pays d'accueil, à la fin des études, particulièrement parmi les étudiants qui ont suivi un parcours recherche, partis sans avoir eu d'expériences professionnelles dans leur pays et qui ont passé plusieurs années en France ou aux États-Unis pour faire un doctorat. Ces personnes ont imaginé des nouveaux projets professionnels pour leur avenir et expriment des réticences pour revenir dans le pays d'origine, réticences qui, avant, n'existaient pas. Une explication facile serait de réduire leurs décisions au volet professionnel. Effectivement, ce groupe d'étudiants a des réseaux professionnels faibles pour envisager une insertion au retour ; cependant, une lecture alternative des discours montre que la longue durée du séjour implique nécessairement une évolution des personnes, de leurs visions du monde et de leurs projets. Après plus de cinq ans de séjour à l'étranger, ces jeunes adultes sont aujourd'hui des chercheurs et ils ont développé un regard critique vis-àvis de leur situation dans leur pays d'origine. Un ingénieur-chercheur colombien, par exemple, m'a expliqué que la sensation de peur avec laquelle il vivait dans sa ville natale, Cali, lui paraissait au moment de l'entretien insupportable, après avoir vécu à l'étranger. Un autre exemple est celui d'Antonia, doctorante chilienne, qui explique qu'une partie de son désir de rester en France est liée à son expérience d'avoir été discriminée au Chili en raison de ses traits indigènes, ce qu'elle n'a, selon ses propres termes, jamais ressenti pendant les sept ans vécus en France. Finalement, il existe plusieurs types de bifurcations et elles se découvrent seulement avec l'expérience de partir et sous un regard attentif à une suite d'évènements. Chercher une meilleure qualité de vie est une quête personnelle et partir étudier à l'étranger est l'un des moyens de quitter ce qu'on n'aime pas et qu'on ne peut pas changer, c'est fabriquer sa propre transformation.

24 - MD. Ceci vaut jusqu'à la vie intime et aux stratégies matrimoniales, semble-t-il, qui constituent finalement aussi une ressource dans les parcours de mobilité sociale?

25 - CPB. Il existe un rapport clair entre modèle familial et appartenance sociale dans la pratique de suivre des études à l'étranger. J'observe qu'une bonne partie des étudiants partent étudier en couple et, justement, les plus aisés sont mariés. Les manières 
d'organiser ce type de séjour d'études ont un rapport avec le profil éducatif des étudiants ainsi qu'avec leurs trajectoires professionnelles. Quand c'est seulement le conjoint masculin qui étudie, il s'agit d'un ingénieur ou d'un économiste ayant travaillé en tant que cadre du secteur public ou privé. En revanche, quand il s'agit d'une femme étudiante et d'un mari qui l'accompagne, elle est diplômée le plus souvent en sciences sociales et a déjà travaillé dans le secteur public ou dans une université du pays d'origine. Enfin, les mariages de deux étudiants sont généralement deux anciens enseignants universitaires dans le pays d'origine. Ces informations permettent de tirer une conclusion : partir étudier à l'étranger en étant marié est une pratique exclusive des jeunes ayant achevé la mise en place d'une situation économique et professionnelle stable dans le pays d'origine. D'autre part, la poursuite d'études à l'étranger participe à la reproduction des élites à la fois mondialisées et conservatrices. Parmi les étudiants interviewés à Boston, dans les universités les plus réputées pour les cadres de direction, la figure du "diplômé international» est ambiguë : très ouverte au monde et très globalisée dans son expérience biographique et professionnelle, elle reste néanmoins conservatrice et attachée aux valeurs traditionnelles familiales et nationales (mariage précoce, femme au foyer, plusieurs enfants, école catholique, rituels propres au pays et enfermement social durant le séjour à l'étranger). Cette vision du monde contribue à désirer le retour au pays d'origine une fois les études achevées. L'éducation des enfants doit éviter les contacts avec une société étrangère qui ne transmet pas cette échelle traditionnelle de valeurs.

Cela dit, bien évidemment, tous les étudiants mariés ne font pas automatiquement partie des élites à la fois mondialisées et traditionnelles. L'étude des différentes modalités d'organisation du séjour d'études à l'étranger en couple met en avant des profils éducatifs et professionnels divers. Parmi les couples mariés, certains appartiennent certes aux classes favorisées mais ne partagent pas forcément la même échelle de valeurs que les plus conservateurs. Pour eux, la division sexuelle du travail n'est pas si marquée, la parentalité est soit reportée à plus tard, soit plus équitablement partagée au cours du séjour à l'étranger. La soumission de la conjointe au projet professionnel de son mari est moins fréquente ; au contraire, les deux poursuivent des études en même temps. Enfin, certains couples sont plus souvent exposés à d'autres milieux sociaux et culturels plus progressistes pendant leur séjour à l'étranger, ce qui les amène à articuler autrement travail et famille au cours du séjour.

Même si d'un point de vue juridique le mariage est indispensable pour la mobilité internationale, le discours des personnes interrogées porte surtout sur la recherche d'une légitimité symbolique. L'engagement prétendument sans réserves qui s'exprime dans la figure du mariage permet aux individus de mieux s'adapter aux projets professionnels du partenaire. Le cas contraire est donc celui des couples qui partent étudier à l'étranger ensemble sans se marier. Eux, à l'inverse des couples mariés, sont sans exception deux étudiants où chacun a son propre projet d'études bien défini. Pour ces étudiants, ainsi que pour les célibataires, partir étudier à l'étranger peut en outre devenir un refuge dans la distance pour se construire plus librement en se libérant des contraintes du passé et du conventionnalisme.

- MD. Pour finir, votre thèse apporte de nombreux éclairages nouveaux permettant de remettre en question certaines idées sur la catégorie des " étudiants internationaux ", qui est présentée dans les politiques migratoires comme une valeur de l'ère néolibérale, autant que sur le « cosmopolitisme ». 
29 sur l'acquisition progressive de cette caractéristique Cet adjectif, que recouvre-t-il exactement? Celui qui vit dans plusieurs espaces et qui navigue d'un pays, d'une entreprise à l'autre comme s'il était chez lui ? Celui qui est revenu au pays fort d'une expérience de quelques années à l'étranger et qui va continuer, sa vie professionnelle durant, à présenter une image marquée par son expérience d'ouverture, de maitrise des langues et des codes, et de réseau (professionnel et amical) international ? Celui qui est resté dans le pays de destination, qui y construit sa vie personnelle, amicale, professionnelle et familiale? Ce dernier ou cette dernière n'est pas perçue comme « internationalisé ». Il parvient à être identifié comme «la francesa » ou « el gringo » pour ses anciens amis du pays, et comme «el chileno» ou «la colombiana » pour ses nouvelles connaissances dans le pays hôte. De plus, la « chilena », ou l'immigrée a dans la société d'accueil une place inférieure à celle de « la francesa » ou l'émigrée au Chili. Les stéréotypes correspondant à la hiérarchisation des pays ont la vie dure, quel que soit le niveau de qualification.

Autant l'internationalisation des étudiants et des professionnels est valorisée dans tous les pays, autant l'immigration continue à être traitée à part, et elle ne parvient pas à supplanter la mobilité temporaire dans l'échelle des valeurs des représentations collectives. Dans ce contexte, la figure du jeune diplôme «internationalisé » suggère l'image d'une mondialisation "post-moderne ", qui se situe bien au-delà des États, où les compétences internationales sont vues comme des valeurs circulantes, débarrassées des ancrages sociaux. Une telle image contribue à négliger l'enjeu national de la prise en charge de la migration en tant que question de société, et à justifier les contrôles sécuritaires et nationalistes de restriction des entrées.

Qui sont les jeunes diplômés « internationalisés »? J’ai suggéré deux visages différents. Le premier tient à la figure du travailleur supposé ne pas connaitre les frontières. Ces jeunes ont un profil très particulier: célibataire, un homme et plus rarement une femme, passionné et très performant, investi dans son activité professionnelle, avec une forte identification à son métier qui représente davantage une vocation qu'une simple occupation professionnelle. Ils rêvent d'une identité tournée vers l'international, de se sentir "citoyens du monde " et ils ont tendance à afficher un léger mépris pour ceux qui ne partagent pas cette philosophie. Contre les conformismes des assignations attendues de stabilisation à l'âge adulte dans des rôles socialement encouragés, ces jeunes adultes affirment au contraire leur jeunesse et une certaine indépendance d'esprit. Le décalage qu'ils constatent avec leurs pairs (collègues et amis des deux pays) apparaît comme une affirmation de soi. Le point de vue est critique sur le pays de départ, notamment sur l'instabilité politique (pour la Colombie) ou le conservatisme (pour le Chili). Ceci n'étant pas partagé par les personnes d'origine sociale très aisée, cela confirme l'hypothèse que l'internationalisation est bien un fait de classe moyenne. Le regard des autres pèse sur les décisions de ces « internationalisés » : ceux qui échappent au modèle savent que cela leur sera reproché à chaque visite au pays. On a donc l'impression que l'internationalisation d'un cursus amorce un regard relativement progressiste, critique et libéré sur certaines échelles de valeurs de la société d'origine. Cependant, il ne faut pas non plus les concevoir comme des anticonformistes car ils finissent par remplacer l'échelle de valeurs locales traditionnelles par une autre, centrée sur la performance, la concurrence internationale et le mythe des citoyens d'un monde hyper-mobile. 
32 Le deuxième visage du jeune diplômé "internationalisé » apparaît chez les professionnels qui retournent dans leur pays après les études. Si la mondialisation devait s'accompagner d'une dé-traditionnalisation, on ne comprendrait pas l'existence de cette figure. Très ouvert au monde dans son expérience biographique, ce professionnel revendique néanmoins les valeurs traditionnelles familiales et nationales (mariage précoce, femme au foyer, plusieurs enfants, école catholique, rituels propres au pays et enfermement social durant le séjour à l'étranger). Cette échelle de valeurs l'amène à souhaiter le retour au pays d'origine où cette vision du monde traditionnelle est présumée être partagée. Finalement, la distinction mise en avant par les élites repose sur l'affirmation de ce qu'on pourrait appeler un «paquet identitaire conservateur ». Le pouvoir économique et politique étant détenu par des milieux fortement marqués par un éthos catholique conservateur, ceux qui souhaitent s'approcher de ce milieu par leur trajectoire professionnelle ascendante s'y conforment, sous peine de ne pas être acceptés. En effet, certains jeunes diplômés ayant tiré de l'expérience internationale une vision critique des valeurs et des usages conformes pourraient faire l'expérience d'un plafond de verre bien particulier : celui de ne pas être en harmonie avec les valeurs des milieux occupant les positions de pouvoir.

Étant donné l'ampleur numérique de l'immigration d'origine latino-américaine aux États-Unis, être chilien ou colombien est loin d'y être exotique. Ce n'est pas pareil en France, où cette immigration n'arrive pas à $10 \%$ du total de la population étrangère. Nombreux ou pas, les émigrants de cette région du monde rejoignent les stéréotypes des travailleurs et familles pauvres des pays du Sud. Dans ce cadre, les discours des étudiants interviewés montrent une mise à distance claire et nette vis-à-vis de la population immigrante de leur même région. Il y a un "nous » composé d'étudiants, chercheurs, professionnels d'origine étrangère, et il y a un «autre » qui réunit les latinos qui habitent durablement dans les pays du Nord. Cette distance est présentée comme une extériorité. La mise à distance des populations immigrantes peut être considérée comme une stratégie - délibérée ou inconsciente - de protection de soi face à la perception d'un regard stigmatisant qui pèse sur toutes les populations étrangères (Sayad, 1999).

\section{BIBLIOGRAPHY}

Bessin M., Bidart C., Grossetti M., 2010. Bifurcations. Les sciences sociales face aux ruptures et à

l'événement. La Découverte.

Granabotter M. S., 1973. The strength of weak ties. American journal of sociology, 78(6),

p. $1360-1380$.

Lahire B., 2005. L'homme pluriel : les ressorts de l'action. Armand Colin.

Passeron J.-C., 1990. Biographies, flux, itinéraires, trajectoires. Revue française de sociologie, 31(1), p. 3-28.

Sayad A., 1999. La double absence. Des illusions de l'émigré aux souffrances de l'immigré. Seuil, Paris. 
Simmel, G., 1979 (1908). Digressions sur l'étranger. In Grafmeyer Y. \& Joseph I, L'École de Chicago, naissance de l'écologie urbaine, Paris, Ed. Du Champ urbain, p. 53-77.

Wagner A. C., 2007. Les classes sociales dans la mondialisation. Paris: La Découverte.

\section{AUTHORS}

\section{CAROLINA PINTO-BALEISAN}

Carolina Pinto-Baleisan, caropintob@gmail.com, est docteure en sociologie de l'Université ParisEst et chercheuse indépendante dans la région de Valparaiso.

\section{MATTHIEU DELAGE}

Matthieu Delage, matthieu.delage@u-pem.fr, est Maître de conférences à l'Université Paris-Est Marne-la-Vallée, Laboratoire ACP. 